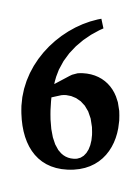

\title{
ALUMNADO INMIGRANTE EN LA ESO: VULNERABILIDAD PEDAGÓGICA DEL SISTEMA EDUCATIVO
}

\author{
(IMMIGRANT STUDENTS IN COMPULSORY SECONDARY EDUCATION: \\ PEDAGOGICAL VULNERABILITY OF THE EDUCATIONAL SYSTEM)
}

Juan Fernández Sierra

Universidad de Almería

DOI: $10.5944 / e d u c X X 1.17494$

\section{Cómo referenciar este artículo/How to reference this article:}

Fernández Sierra, J. (2017). Alumnado inmigrante en la ESO: Vulnerabilidad pedagógica del sistema educativo. Educación XX1, 20(1), 121-140, doi: 10.5944/educXX1.17494

Fernández Sierra, J. (2017). Alumnado inmigrante en la ESO. Vulnerabilidad pedagógica del sistema educativo. [Immigrant students in compulsory Secondary Education: Pedagogical vulnerability of the educational system]. Educación XX1, 20(1), 121-140, doi: 10.5944/educXX1.17494

\section{RESUMEN}

Es preocupante el alto porcentaje de alumnado inmigrante que no culmina con éxito la enseñanza obligatoria, extendiéndose una tendencia de pensamiento docente que identifica las causas con cuestiones de índole personal, sociofamiliar o cultural. Sin embargo, parece razonable indagar en qué yerra nuestro sistema educativo que no logra superar esa situación. En esa línea, el objetivo esencial de esta investigación ha sido indagar en los puntos débiles o vulnerabilidad del sistema (Reimer, 1973) para responder a este escenario, enfocándolo desde la perspectiva de los planteamientos de política curricular y examinando las raíces institucionales y pedagógicas que inciden en la deserción y fracaso escolar. Para ello, hemos realizado un diseño de corte naturalista basado en ocho estudios instrumentales de caso, llevados a cabo en otros tantos institutos de diversas características, desarrollándolos al modo de lo que Stake (2007) denomina estudio colectivo de casos.

En cuanto a los resultados y conclusiones, damos especial relevancia al tándem formado por la que podríamos denominar vulnerabilidad intrínseca —actitudes y prácticas hegemónicas mediatizadas por la cultura profesional 
instalada en este nivel educativo- y la vulnerabilidad estructural — políticas educativas y curriculares-, que se retroalimentan mutuamente, construyendo una resistencia simbólica, normativa y de práctica pedagógica, inhibidora de los intentos innovadores para reorientar la acción educativa hacia dimensiones más inclusivas, multiculturales e interculturales. Los estereotipos y la cultura académica previa experimentada por las familias y prole, también se muestran como factores significativos de vulnerabilidad. El diseño empleado, por su propia naturaleza, no permite generalizar los hallazgos, pero sí posee un alto poder de transferibilidad y de aportación de interpretaciones y asertos para seguir comprendiendo las complejidades de la educación actual e ilustrar la toma de decisiones políticas y prácticas en este ámbito.

\section{PALABRAS CLAVE}

Educación obligatoria; multiculturalidad; vulnerabilidad; política curricular; inmigración; cultura escolar.

\section{ABSTRACT}

The high percentage of immigrant students who do not successfully complete compulsory education is of great concern, as is the increasing trend among teachers of ascribing this failure to personal, social, family or cultural factors. Nevertheless, the potential deficiencies of the educational system for addressing this situation should also be investigated. Thus, the main aim of this study was to identify the weaknesses or vulnerabilities of the system (Reimer, 1973) for responding to this situation, focusing on curriculum policy approaches and examining the institutional and educational roots of school dropout and school failure. The study had a naturalistic design based on eight instrumental case studies carried out in eight different schools and was developed according to what Stake (2007) called a collective case study.

The results lead us to place special emphasis on what we call intrinsic vulnerability (dominant attitudes and practices mediated by the entrenched professional culture at this level) and structural vulnerability (educational and curriculum policies). Through mutual feedback, these vulnerabilities create symbolic and regulatory resistance as well as resistance in pedagogic practice, hindering innovative attempts to reorient educational action towards more inclusive, multicultural, and intercultural dimensions. Both stereotyping and the previous educational culture experienced by families and children are also important vulnerability factors. Due to the intrinsic design of the study, the findings cannot be generalized; however, it is highly transferable, and may provide interpretations and assertions that will continue to deepen our understanding of the complexities of the current education system and shed light on decision-making regarding policies and practices in this area. 


\section{KEYWORDS}

Secondary School Curriculum; Multicultural Education; Educational Inequality; Educational Policy; Migrant Education; School Culture.

\section{INTRODUCCIÓN}

No existe ningún niño que no aprenda algo de la escuela. Los que nunca pueden ingresar a ella, aprenden que las cosas buenas de la vida no les corresponden... Los que desertan..., aprenden que el sistema es vulnerable, aunque no sean ellos quienes puedan golpearlo (Reimer, 1973, p. 4).

En las sociedades avanzadas, la mayor parte de la población se considera integrada o incluida, en el sentido que participan, disfrutan y viven en un estado aceptable de bienestar social, personal, grupal, familiar, económico y asistencial. No obstante, hallamos minorías más o menos amplias que, por el contrario, quedan «atrapadas» en ese indeseable status que supone estar «fuera de». El camino de la inclusión hacia la exclusión está lleno de circunstancias, factores y variables diversas, normalmente ligadas a condiciones económicas, a las que se le suman otros motivos como la salud, el paro o el déficit educativo. En relación a este último aspecto, una de las funciones primordiales del sistema de enseñanzas obligatorias es tratar de interponerse, actuando pedagógicamente con todos los niños, niñas y jóvenes, para que no crucen ese umbral excluyente y, en su caso, ayudarles a recuperar el camino hacia la inclusión. Sin embargo, en nuestro país, en 2005, el 48,8\% de los alumnos extranjeros habían «sufrido la vulnerabilidad» de la que hablaba Reimer (1973), y en 2011 seguían siendo el 43,9\% (CC.OO., 2013). Se hace preciso, pues, indagar en qué aspectos yerra o presenta vulnerabilidad nuestro sistema educativo - ese ha sido el propósito de este estudio-, que no logra alejar de esta zona de exclusión a una gran parte de los hijos e hijas de inmigrantes incorporados a él.

\section{LA VULNERABILIDAD EN EDUCACIÓN}

La propia definición que la Real Academia Española otorga a la palabra vulnerable — «que puede ser herido o recibir lesión, física o moralmente» (RAE, 2001)_, refleja la diversidad de órdenes y campos en los que es susceptible de aplicarse. La utilización más extendida y consensuada del término es aquella que incluye tanto los riesgos, contingencias y presiones que puede experimentar una determinada población o grupo humano ante cualquier tipo de catástrofe, como la dificultad, indefensión o falta de 
medios por parte de dicho colectivo para afrontarla y reponerse ante ella (Turner et al., 2003). Hasta mediados del siglo pasado, el discurso sobre la vulnerabilidad se enfocaba desde una perspectiva de excepcionalidad y eventualidad de los acontecimientos «naturales», sin relación causal con los tejidos y elementos sociales. Fue a partir de la década de 1970 cuando comienza a desarrollarse una concepción de carácter más social, con el punto de mira en los procesos y sistemas socioeconómicos como principales propiciadores de los desastres (O»Keefe, Wetsgate \& Wisner, 1976; Pérez de Armiño, 2000).

Desde el ámbito de la sociología, el concepto de vulnerabilidad se ha trabajado en estrecha relación con el de "exclusión», siendo la concepción más extendida la que la define como la situación de aquellos individuos o colectivos ubicados en una zona intermedia permanentemente inestable entre la integración y la exclusión, entendidas ambas como extremos opuestos en el grado de participación en la sociedad (Castel, 1995, 2007), siendo los motivos fundamentales para caer en la exclusión la acumulación de barreras o riesgos asociados a las condiciones laborales, económicas, socio-sanitarias y formativas, así como la dificultad de acceso a los diferentes mecanismos de protección necesarios (Subirats Humet, Gomà Carmona \& Brugué Torruella, 2005; Planella Rivera, 2008). En el campo de la educación, especialmente en países en desarrollo, se realizan numerosos estudios midiendo diversos factores como el analfabetismo, el fracaso escolar o el nivel de formación, relacionándolos con la vulnerabilidad de los colectivos.

Este trabajo no lo enfocamos desde esa perspectiva de «situaciones educativas» como indicadores de vulnerabilidad social, sino que hemos partido de la premisa de que ningún niño o niña que asista a la escuela (como sucede en España) debiera clasificarse, en principio, como "excluido» por motivos educativos, independientemente de los factores o fuerzas centrífugas concurrentes en los que se desenvuelva su vida; pero sí hemos de considerar a muchos de ellos y ellas ubicados en zonas de vulnerabilidad más o menos resbaladizas, en las que una formación adecuada debería situarlos en zona de inserción (Tezanos, 2001) y ser un factor de re-inclusión (Vélaz de Medrano, 2005; Fernández Sierra, 2006). Esto requiere que la educación obligatoria sea un elemento eficaz de resistencia y rescate ante la exclusión; aunque los datos nos indican que eso no se produce en la proporción deseada (Roca Cobo, 2010; Aguado Odina \& Grañeras Pastraña, 2010; MECD, 2012), por ello hablamos de «vulnerabilidad pedagógica», o sea, de la existencia o concurrencia de factores, circunstancias y fenómenos de diverso tipo, arraigados en las políticas y prácticas educativas, que dificultan y suponen fragilidad para la posibilidad y futuro educativo de colectivos o personas determinados, como los jóvenes inmigrantes. Una concepción en línea con trabajos como los de Escudero Muñoz (2005) o Mena Martínez, 
Fernández Enguita \& Riviére Gómez (2010), que instan a considerar y analizar las raíces institucionales y pedagógicas - no solo las sociales y personales- a la hora de abordar el fenómeno de la deserción y fracaso escolar.

\section{METODOLOGÍA}

Los objetivos esenciales de la investigación aquí presentada han sido indagar en las dificultades o carencias en los activos y recursos simbólicos requeridos para que los chicos y chicas inmigrantes puedan aprovechar las oportunidades del sistema educativo, relacionándolos con los puntos débiles o vulnerabilidad de este sistema para responder a las desventajas que pudiesen acompañarles.

El artículo está basado en ocho estudios instrumentales de caso -llevados a cabo en otros tantos centros de la Comunidad autónoma andaluza en los que se imparte el primer ciclo de Educación Secundaria Obligatoria-. Casos que, siguiendo a Stake (2007), nos dan la oportunidad de ir más allá de la comprensión de las situaciones singulares seleccionadas, para poder utilizarlos como elementos o artefactos que nos sirvan para aprender sobre la problemática con la que estos chicos y chicas se topan en el sistema de enseñanza, así como las complejidades a las que dicho sistema se enfrenta para responder adecuadamente a su educación.

Los centros fueron seleccionados en razón a: ciclos o etapas que abarcan - CEIP con primer ciclo de ESO; IES con solo ESO; IES con Bachillerato; IES con ciclos formativos-, y enclave socio-geográfico rural/urbano. Todos ubicados en zonas de alta concentración de población inmigrante, con más del 10\% de alumnado extranjero en el primer ciclo de la ESO.

Las técnicas de recogida de datos - realizada durante dos cursos escolares de trabajo de campo- han sido: observación participante en las aulas, espacios comunes y recreos; conversaciones informales con alumnado y docentes; entrevistas en profundidad a estudiantes, profesorado y familiares cercanos, y análisis de documentos institucionales y académicos. La información recogida mediante dichas estrategias, se ha coordinado y contrastado con reuniones periódicas de los investigadores/as de cada caso - a modo de lo que Stake (2007, p. 17) llama "estudio colectivo de casos» complementándose y validándose con dos grupos de discusión (Barbour, 2013), en los que han estado representados diversos profesionales de los centros, responsables de la Administración, estudiantes de diferentes procedencias y familiares de alumnado de origen inmigrante ${ }^{1}$. La credibilidad del estudio queda avalada por el trabajo de campo prolongado en los centros (dos cursos); la triangulación de los métodos aludidos y de los investigado- 
res de los distintos casos, y el contraste de los datos con los participantes (representados en los dos grupos de discusión).

\section{RESULTADOS}

En concordancia con los objetivos y metodología expresados, la codificación, segmentación y reorganización de las informaciones recogidas han alumbrado una serie de categorías que tomamos como base para la estructuración y exposición que aquí hacemos, representando cada una de ellas un elemento con suficiente potencial explicativo para acercarnos a la comprensión de la vulnerabilidad del sistema ante este sector del alumnado, a saber: a) las culturas docentes hegemónicas, b) las prácticas didácticas, c) la cultura y experiencia académica previa de los estudiantes y sus familias, d) los estereotipos del grupo de pares y e) las políticas educativas y curriculares vigentes.

\section{Las culturas profesionales: vulnerabilidad conceptual}

Con la expresión vulnerabilidad conceptual nos referimos a la dificultad de muchos docentes para replantearse su labor, «atrapados» en su acervo formativo y la cultura profesional heredada. Los modos de acción pedagógica dependen del pensamiento práctico de los docentes, de sus creencias, representaciones y expectativas (Gimeno Sacristán \& Pérez Gómez, 2008; Contreras Domingo, 2011; Hargreaves \& Fullan, 2012), mediatizadas, cuando se trata de alumnado extranjero, por su ideología personal/profesional sobre la inmigración. En este aspecto, numerosos estudios muestran cómo en función de ello, el profesorado establece diferentes compromisos y estilos de relación con este alumnado (Defensor del Pueblo, 2003; Essomba, 2006; Colectivo IOE, 2007; Leiva Olivencia, 2011). En nuestro estudio hemos evidenciado que, cuando se incorporan a estas clases jóvenes inmigrantes, aparecen posicionamientos pedagógicos y modos de actuar en tres líneas diferentes.

Por un lado, una minoría -especialmente significativa y distorsionadora de alternativas pedagógicas-, que considera una contrariedad y complicación la incorporación de estos estudiantes, lamentándose de la disrupción que les supone el que se les asignen algunos de ellos a su clase, manifestando que la educación de este alumnado no debería ser de su responsabilidad profesional — «...yo soy profesor de Ciencias, para eso me preparé, no para aguantar a gente que no se entera de lo que explico" (Cas.3-profe)—. En estos casos, las aulas pasan de ser lugares de inclusión a fuerzas centrífugas hacia la deserción: «Ante la llegada de estos alumnos al 
centro no se planteó nada, e incluso algunos compañeros, cuando hablaban de ellos, les llamaban «moros»...» (gru.dis-profa). Alegan estos docentes que los alumnos no están capacitados para seguir los niveles académicos exigidos y procuran que estén fuera de clase el mayor tiempo posible -en grupos específicos, en clases de apoyo o fuera-: "Cada vez que incordian los mando con el orientador o a la jefatura de estudio» (Cas.2-profe). Durante el tiempo que obligatoriamente han de estar en el espacio físico del aula ordinaria, los mantienen pasivos, observándose en algunos casos actitudes poco educativas: «¿Habmeth?, ahi está, rellenando los cuadernillos que le ha dado la orientadora, siempre que no interrumpa...» (Cas.8-profe).

Otro sector, el mayoritario, tiene asumido un estilo docente y una concepción neoclásica propedéutica de la enseñanza obligatoria y de su función profesional, que les predispone a percibir el hecho migratorio como un problema eminentemente instructivo. Son docentes convencidos de la importancia del conocimiento disciplinar y de los rendimientos académicos, sintiéndose a menudo desbordados: "No es que no quiera, es que no sé, no puedo atender a todos, con tantos niveles» (Cas.6-profa). Ven el gran problema en el idioma y han internalizado una concepción intrapsíquica de las responsabilidades sobre el aprendizaje que les provoca la invisibilidad o negación de las peculiaridades de este alumnado, lo que dificulta actuaciones educativas singulares: "Para mí los alumnos inmigrantes no suponen ningún problema, siempre que tengan superado el idioma. Una vez superado esto no hay diferencias, el que quiere estudia y el que no, ¡como los españoles!» (Cas.7-profe).

En tercer lugar, encontramos una minoría - activa y reconocida en los centros, pero poco apoyada en sus propuestas pedagógicas-que interpreta la incorporación del alumnado inmigrante como una nueva situación socioeducativa a la que el sistema de enseñanza ha de dar respuesta desde una visión inclusiva, respetuosa con la diversidad, que aporta riqueza cultural y nuevos retos docentes. Son profesionales que intentan innovar y renovar la educación, no solo para los y las hijas de inmigrantes, sino para adecuarla a todos y cada uno de los niños y niñas: "Somos los profesores los que tenemos que coger el toro por los cuernos. Lo que sí sería interesante es el intercambio de experiencias» (Cas.4-profa).

\section{Las prácticas docentes transmisivas: vulnerabilidad didáctica}

En función de su pensamiento práctico, el profesorado pone en escena actuaciones y estrategias docentes distintas para responder a esta nueva situación, como fruto de sus particulares concepciones educativas y de su visión sobre las especificidades socioculturales de estos chicos y chicas. El 
efecto no deseado de sus prácticas en el aula, que puede operar como fuerza centrífuga con determinado alumnado, es lo que denomino vulnerabilidad didáctica.

Lo primero a destacar, en este aspecto, es el generalizado enfoque homogeneizador impersonal de la acción docente, íntimamente unido a una concepción unidireccional de los procesos de enseñanza-aprendizaje. Modelo individualizado e individualista que no garantiza la comunicación intercultural, el intercambio, la aproximación y el reconocimiento del otro. Cuando se dice querer adecuar las enseñanzas a las singularidades de estos chicos y chicas, suele hacerse desde un instrumentalismo utilitarista que limita aún más la adaptación personal: «Lo principal es que aprovechen aprendiendo lo mejor que puedan a leer y a escribir el español... muchos llegan ya mayores y como van a estar poco tiempo en la escuela, no van a tener muchas oportunidades»(Cas.1-profe).

El concepto de inclusión de estos profesionales, entendido casi exclusivamente en torno al dominio de contenidos académicos, potencia la organización disgregadora de este alumnado, visualizada mediante tres estrategias didácticas predominantes en los centros: la segregación del aula ordinaria para el aprendizaje del idioma, el apoyo extra-aula para la enseñanza de las materias consideradas básicas y la preponderancia de tareas paralelas desconectadas en la propia clase. Este proceder, en gran parte justificado bajo el paraguas de la atención lingüística (Vila, 2005), presenta grandes limitaciones y se hace cada vez más insostenible y menos eficaz conforme va aumentando y estabilizándose el número de hijos e hijas de inmigrantes y avanzamos hacia una sociedad más multicultural.

Modo de actuar en el que concurren diferentes especialistas — docentes, orientadores, apoyos y mediadores-que podría ser en algunos aspectos eficaz si se planificara y pusieran en práctica actuaciones multiprofesionales cooperativas (Fernández Larragueta \& Rodorigo, 2012). Sin embargo, nuestro trabajo muestra una evidente descoordinación de las acciones compensatorias respecto a la estructura de tareas académicas y del sistema de relaciones sociales del aula. Estos chicos y chicas reciben generalmente ayuda complementaria para el aprendizaje del español y clases de recuperación sobre materias básicas, pero al realizarse extra-aula, se revelan poco exitosas, descolgándoles de sus grupos-clase, dificultándoles su participación e inserción; forzándolos hacia un "currículo paralelo» asistemático y accidental: "Cuando voy a clase de ATAL, me pierdo la clase, y ya me he perdido lo que han explicado, cuando preguntan lo que se explicó ese día, yo no lo sé»(Cas.2-alumno). 
Lo más preocupante y erróneo de esta pedagogía es que, en numerosas ocasiones, cuando permanecen en el aula ordinaria, están realizando tareas sin relación con el trabajo del resto de compañeros: "En Lengua a veces le doy fichas, por lo menos que trabaje, porque lo del libro de texto sé que no se lo va a aprender» (Cas.7-profa). Estrategia que potencia muchas veces el aislamiento y la creación de grupos cerrados y desubicados, estigmatizando al que va a la recuperación:

Cuando llego a la clase, todos los alumnos, menos Yakima - alumna marroquí-, están preparando los trabajos manuales que se venderán en el mercadillo que han organizado para el Día de la Paz. Ella no participa. Está esperando que venga a buscarla el profesor que le da apoyo en materias básicas (Cas.4-invg).

La clave que sustenta este arco didáctico centrífugo es la presión evaluativa basada en contenidos disciplinares eminentemente memorísticos. La enseñanza fundamentada en la retentiva y reproducción de las síntesis de los libros de texto supone un esfuerzo extra para los estudiantes emigrantes, impidiéndoles dedicar su tiempo escolar a la adquisición de aprendizajes significativos y relevantes, colaborando a su baja formación y desalentándoles en sus expectativas académicas y de futuro:

Cuando toca examen tengo que estudiar mucho tiempo... los españoles solo lo leen unas pocas veces y pueden escribir con sus palabras, nosotros estudiamos más horas para escribir exactamente bien las respuestas. Tengo buena memoria, pero solo si dedico mucho tiempo para memorizar (Cas.5-alumna).

\section{La cultura académica previa: vulnerabilidad experiencial}

En los estudios sobre fracaso o rendimiento escolares, aparece siempre como factor determinante el nivel sociocultural de las familias (Marí-Klose, 2009; OCDE, 2013); así pues, si partimos de la constatación de que dicho nivel en los inmigrantes de nuestro entorno es medio-bajo o bajo, encontramos un factor de vulnerabilidad evidente. No obstante, en este estudio, este componente por sí solo no explica las dificultades escolares de los chicos y chicas inmigrantes, sino que la incidencia mayor viene mediatizada por la cultura académica vivida, en el sentido que la entiende Pérez Gómez (1998, p. 253), «como la selección de contenidos destilados de la cultura pública para su trabajo en la escuela: el conjunto de significados y comportamientos cuyo aprendizaje se pretende provocar en las nuevas generaciones a través de la institución escolar»; cuestión relacionada con lo anterior, pero no idéntica. La incidencia del acervo académico de origen de estos estudiantes y sus familias, la analizamos bajo el epígrafe de vulnerabilidad experiencial. 
Los grupos de inmigración en los centros estudiados -magrebíes, europeos del Este e iberoamericanos- presentan, cuando se incorporan a la escuela, diferencias formativas y simbólicas. Los primeros no tienen el español como lengua materna y sus valores ideológico-religiosos se ubican en la órbita musulmana; los segundos tampoco hablan nuestro idioma; mientras que los terceros comparten lengua, considerándose partícipes del pensamiento occidental. Las creencias asentadas tienden a deducir que sus dificultades para la integración o inserción en el sistema educativo se darían en el orden en el que han sido enunciados. Pero ese marco es genérico y difuso, de modo que junto a él encontramos un aspecto que se revela como un factor potente para explicar las dificultades curriculares de estos estudiantes: el grado de similitud de la cultura académica experimentada por ellos y sus familias en sus países de origen, respecto a la de acogida.

En referencia al alumnado procedente de los países del Este, muchos docentes tienden a asignarle ciertas cualidades intrínsecas, considerándolos más inteligentes y formados que los de otros orígenes - "a los del Este no les cuesta nada, son hábiles intelectualmente y gozan de una historia escolar, con sistemas de autoridad, disciplina, etc.»(Cas.1-profa)—; sin embargo, no es razonable esa recurrencia un tanto innatista, sino que el relativo éxito adaptativo está relacionado con su socialización socio-escolar previa. Es decir, los sistemas educativos de dichos países - a los que han asistido los padres y madres ahora aquí inmigrados-, presentan alta similitud con el nuestro en cuanto que han potenciado una cultura escolar academicista y un currículum por disciplinas. Podríamos decir que la mayoría de los estudiantes y sus familias vienen con «las reglas del juego escolar aprendidas», indistintamente de su nivel económico y sociocultural. Esto a menudo se convierte en una ventaja escolar: "Se adaptan mejor al ritmo de clase..., el alumnado que procede de países del Este es disciplinado; está la barrera idiomática, pero aprenden rápidamente el castellano, por lo que se pueden incorporar más rápido al ritmo del curso» (gru.dis-profa).

Las experiencias de las chicas y chicos iberoamericanos, en cuanto a sus procesos de escolarización previos, son muy variadas, pero en general sus sistemas educativos se caracterizan por ser elitistas y poco eficaces (Espínola Hoffmann \& Claro Stuardo, 2010), aunque con gran variedad de unos países a otros y, sobre todo, con grandes diferencias entre clases sociales y familias. Esto tiene como consecuencia que los estudiantes de esta procedencia -mayoritariamente emigrantes económicos con diferentes niveles formativos-, suelen carecer de una experiencia escolar de partida aceptable, aunque sus padres valoran la educación como elemento importante para la promoción social: "Yo quiero que estudien, yo no pude estudiar... Yo les digo, ustedes tienen la oportunidad de estudiar, quiero que tengan un futuro, que ellos digan qué les gustaría ser»(Cas.2-fami). Los currículos es- 
colares de estos países son, en su diseño, bastante semejantes al nuestro. En principio, podrían dominar las «reglas del juego escolar», pero solo aquellas y aquellos cuyas familias y ellos mismos tuvieron oportunidad de una escolarización adecuada, las comprenden realmente: "Habría que saber de qué parte es, porque aunque sabe el castellano, es como si a ella le costara todo un poco más»(Cas.6-profa).

El tercer grupo sociocultural mencionado —el alumnado magrebí- es más variado y complejo en cuanto a su experiencia escolar, por las peculiaridades tan heterogéneas de sus lugares de procedencia y contextos sociofamiliares; sin embargo se tiende a representarlos de manera más uniforme. $\mathrm{Y}$ en esa creencia homogeneizadora encontramos un importante elemento de vulnerabilidad, pues con esta idea social, compartida por muchos docentes, se invisibiliza a las personas y se les disuelve en un grupo que, por otra parte, se hace indefinido, desconocido y representado simbólicamente por características mayoritariamente negativas.

\section{El contexto sociocultural y los estereotipos: vulnerabilidad frente a los pares}

Los niños y niñas autóctonos comienzan la estructuración de relaciones sociales en el centro educativo a edad muy temprana y, en gran parte, dentro de su ámbito sociocultural próximo. Los y las inmigrantes realizan este tránsito en distintas edades, momentos, períodos, ambientes, etc., lo que les sitúa, en principio, en condición de desventaja inclusiva (Alegre Canos, 2008), ya que, al estar el grupo constituido de años anteriores, se encuentran relativamente asentadas las redes de comunicación, poder, relación y emocionales: "Esmeralda se incorpora al centro semanas después de haber empezado el curso. Se sienta al lado de otros dos compañeros, también nuevos en el grupo-clase. Se relaciona casi exclusivamente con su nueva compañera de origen marroquì" (Cas.3-invg).

El recién llegado, de alguna manera, pone en movimiento dichas redes, las cuales es necesario ir recomponiendo para establecer una comunicación didáctica y personal que permita el avance del aprendizaje del grupo y de cada uno de sus componentes, con especial esmero y miramiento al nuevo incorporado. Las resistencias surgen espontáneamente, la capacidad y habilidad pedagógica del profesorado es puesta a prueba constantemente y su acción certera es la puerta de la inclusión. En este proceso, observamos cómo las representaciones de los «asentados» sobre los advenedizos, o sea, los estereotipos preconcebidos del alumnado que conforma el grupo-clase, marcan la comunicación intercultural. 
Cuando el chico o chica es inmigrante, se evidencian una serie de rasgos físicos y simbólicos que juegan un papel esencial en las concepciones, apreciaciones, actuaciones y expectativas del grupo y de cada uno de sus individuos, incluido el docente. En este sentido, hemos constatado cómo muchos niños y niñas tienen formadas representaciones simbólicas más o menos discriminatorias sobre la procedencia, cultura, religión, bondad o higiene de estos chicos y chicas; es lo que llamamos vulnerabilidad frente a los pares. Los prejuicios pueden observarse en bromas o frases dirigidas hacia ellos: "Me has tocado. Mira cómo me has puesto (simula mancharse porque el compañero es negro)» (Cas.5-alumno); "Qué mal huele por aquí» (ante la presencia de una compañera magrebí)»(Cas.4-alumno). Manifestaciones que pueden adquirir un tono xenófobo e incluso racista preocupante:

Invg: En la clase sobre la inmigración he visto que participas bastante, ¿qué opinas de ese tema?

Alumno: "iSiempre la inmigración!, porque los moros no me gustan. Me dan miedo. No quiero que lleguen aquí. Si no tienen dinero que se queden en su país»

Invg: ¿Tú conoces algún magrebí?

Alumno: «Sí, hay muchos en mi barrio, una vez se metieron con amigos míos, pero a mí nunca me han hecho nada»

Invg: ¿Otras personas también te dan miedo?

Alumno: "Los negros no todos me dan miedo, los que no conozco, tengo un amigo negro, juega a baloncesto, ese no me da miedo, es mi amigo» (Cas.5-invg).

Especial encono se evidencia, a veces, en las relaciones de género, pues el peso xenófobo y machista del ambiente social a menudo hace mella, como nos explica esta profesora: "En el centro, las niñas reciben insultos hasta de los niños de su etnia... son lo último. Un niño, por obtener el reconocimiento de su grupo, es capaz de molestar o insultar a otras de su etnia o a sus hermanas» (gru.dis-profa).

\section{La política educativa y curricular: vulnerabilidad estructural}

El quinto bloque, aquí considerado, de fuerzas centrífugas hacia la exclusión educativa o, al menos, limitadoras de la eficacia inclusiva de los hijos e hijas de inmigrantes — analizada bajo la categoría vulnerabilidad es- 
tructural—, viene por vía de la política educativa gerencialista institucionalizada históricamente en nuestro país. En las últimas décadas se ha realizado un gran esfuerzo para adecuar el sistema educativo a una sociedad democrática, económica y culturalmente en rápida evolución: se fue ampliando la escolarización obligatoria, introduciendo la comprehensividad, integrando a los que hasta entonces se habían catalogado como «deficientes», bajo el lema «todos iguales, todos diferentes», etc. Pero, en «los diferentes», no se preveía a «los otros», a los hijos e hijas de inmigrantes. El sistema educativo se encuentra con dificultades para reaccionar, evidenciándose desorientación y limitaciones para responder de forma adecuada a la nueva situación.

El primer factor limitante, en este aspecto, es la persistencia política de organizar el currículo parceladamente, en su diseño y en su ejecución, con modelos en los que tiene poca cabida la multiculturalidad, la interculturalidad ni la interdisciplinariedad (Torres Santomé, 2008) lo que coarta las posibilidades de innovación y contextualización de las acciones singulares que requiere la diversidad de este alumnado. El problema no es nuevo: los fracasos, abandonos y menores rendimientos se acumulan tradicionalmente en las clases socioculturales menos favorecidas. La incorporación de niños y niñas inmigrantes solo ha puesto el dedo en la llaga, pues al introducirse elementos culturales diferenciados más tangibles o directamente observables, se hacen más evidentes y visibles las contradicciones y limitaciones del currículo:

Damos música clásica... en mi país hay compositores importantes que he investigado con mis padres, pero cuando los digo, el profesor me dice que esos no son importantes, que hay que estudiar los que están en el libro de texto (Cas.2-alumna).

Los gobiernos, siguiendo la tradicional propensión a legislar más allá de los marcos necesarios para organizar el sistema educativo, pretendiendo controlar la actuación docente y construir la teoría y la práctica pedagógica desde el BOE, producen gran cantidad y variedad de normativas con poca incidencia en la práctica cotidiana del aula. Esto, independientemente del análisis que de cada ley o norma hagamos, supone un freno a la búsqueda y puesta en escena de proyectos educativos contextualizados adecuados a las características y necesidades sociales y personales de estos jóvenes. Forma de actuar que ha ido des-profesionalizando la teoría y la práctica pedagógica en nuestro país, afianzándose la idea de que los problemas educativos son eminentemente asuntos administrativo-políticos, transmitiéndose un modo de entender los cambios y las mejoras verticalmente, como una responsabilidad casi exclusiva del gobierno, obviándose la implicación social y profesional que ello requiere. Eso ha derivado en una "cascada evasiva» en la que se van eludiendo y delegando las responsabilidades de los fracasos desde la 
Administración al profesorado y de este a los y las estudiantes y familias: "La solución parte de las casas, de las familias inmigrantes... que deberían tomarse la educación un poquito más en serio...»(Cas.6-profe).

En línea concurrente, los debates políticos sobre educación se trivializan, centrándose reiteradamente en asuntos formales y altamente ideologizados de forma partidista, obviándose las dimensiones sustantiva, social y científico-técnica de la acción docente. Ello está promoviendo un descrédito progresivo del sistema de enseñanza y una desidia e incredulidad respecto a sus perspectivas de futuro y su capacidad de respuesta a las necesidades educativas actuales. En el terreno práctico potencia que numerosos profesionales, unos desilusionados y otros por comodidad, eludan la parte de responsabilidad que les corresponde en cuanto a su formación científico-pedagógica, toma de posiciones profesionales e implicación socioeducativa.

Este círculo de vulnerabilidad, potenciado por la presión productivista y la exigencia de rendimientos sin cambiar el currículum (Fernández Sierra, 2011), se estrecha con la débil comprensividad de nuestro sistema educativo, en el que se potencia y ejerce una actuación organizativa en los centros, selectiva o clasificatoria, que da lugar a estrategias diabólicas para sortear la normativa, como lamenta esta orientadora:

Los grupos de $1 .^{\circ}$ de ESO se organizan en base a su elección o no de la asignatura de Religión... los que no cursan Religión Católica los meten en una clase determinada, entonces hay clases en las que la mayoría son de origen inmigrante, y todo lo que se hubiera conseguido en la escuela respecto a integración, en el instituto se difumina (Cas.2-orient).

\section{CONCLUSIONES}

El alto porcentaje de alumnado de origen inmigrante que no culmina con éxito la educación obligatoria, casi duplicando al de estudiantes españoles, ya de por sí demasiado elevado - $45 » 8 \%$ de abandono prematuro de inmigrantes y $26 » 4 \%$ de autóctonos (Ivie \& Bancaja, 2011) - es una preocupación generalizada en el ámbito de la práctica y política educativa en nuestro país. Los estudios nacionales e internacionales muestran cómo los abandonos y fracasos escolares se correlacionan especialmente con el nivel sociocultural de las familias, dato que nos reitera uno de los puntos débiles de nuestro sistema, pero que al mismo tiempo puede servir de justificación o atenuante, de manera más o menos consciente o subyacente, para los diferentes responsables de la educación. 
La incorporación de alumnado inmigrante a la ESO, con idiomas maternos diferentes, distintas pautas conductuales, variados sistemas de valores y disímiles experiencias académicas, tanto suyas como de sus progenitores, ha cambiado sustancialmente el contexto de actuación docente. Sin embargo, en los casos estudiados, la mayoría del profesorado de este nivel no alcanza a comprender la importancia o incidencia de estos factores en las posibilidades formativas de sus estudiantes (más allá del dominio o no del idioma) ni a tomarlas en consideración a la hora de gestionar el aula y planificar las enseñanzas y estrategias didácticas para llevarlas a efecto. En esta línea, hemos constatado la tendencia de estos docentes a justificar y asumir que, dado el contexto cultural y familiar mayoritariamente precario de esos chicos y chicas, los resultados desfavorables son difícilmente cambiables.

La llegada de estos chicos y chicas conlleva otra dimensión importante: la reestructuración de las relaciones sociales del aula, nicho ecológico donde se despliegan, establecen y desarrollan los procesos de enseñanzaaprendizaje. Aquí, como se ha visto en otros trabajos (Alegre Ricard \& González Motos, 2012), hemos evidenciado la entrada en escena de variables tan influyentes y distorsionadoras como los estereotipos o la xenofobia. La atención a estas relaciones y a la comunicación intercultural se tiene poco en cuenta por parte del profesorado, primándose las cuestiones referidas a los contenidos, dándosele mayor importancia a la «integración» del y la adolescente inmigrante dentro del entorno curricular-académico que a su particular aclimatación e inclusión en el contexto socioescolar. La acción docente permanece asentada en un pensamiento práctico tradicional transmisivo, con praxis didácticas poco concordantes con una educación para la era digital (Pérez Gómez, 2012).

El sistema tampoco acaba de asumir que ya no es el mismo; pues desde el momento en que se incorporan otras culturas, otras experiencias escolares, otros estudiantes y familias, sufre una significativa reestructuración, más o menos visible; sin embargo, parece aferrarse a similares diseños organizativo-pedagógicos a los que están produciendo el alto fracaso. Cuando aumentan las dificultades, se intentan recuperar modos de acción didáctica y organización curricular desfasadas o atemporales, diseñadas para modelos monoculturales homogeneizadores. De esta manera, estos chicos y chicas se topan con ambientes académicos que les son extraños, no solo como efecto del desplazamiento de su país, sino porque además, muchos de ellos y sus familias, no dominan, ni comprenden, ni poseen experiencias ni representaciones simbólicas sobre la escolarización, que les sean útiles para manejarse en este contexto.

Así pues, lo que hemos llamado vulnerabilidad estructural de nuestro sistema de enseñanza obligatoria, junto a las actitudes y las prácticas 
profesionales hegemónicas, forman un tándem que se retroalimenta mutuamente, construyendo una resistencia simbólica, normativa y de práctica pedagógica, limitadora e inhibidora de los intentos para reorientar la acción educativa hacia dimensiones más inclusivas e interculturales, acordes con la presencia de estos estudiantes en aulas sin tradición multicultural. La formación especializada por materias y academicista del profesorado de Secundaria encuentra el terreno abonado para el «no cambio», al sentirse identificado con la organización disciplinar del currículum oficial y los discursos sobre calidad como sinónimo de rendimientos; atrapado — como diría Escudero Muñoz (2009) — en la tensión entre los ambiciosos objetivos de esta etapa y la prosaica realidad de la cultura profesional heredada o sobrevenida. En esta línea, opinamos que las políticas curriculares que se basen solamente en el aumento del logro de rendimientos disciplinares están llamadas al fracaso porque, según hemos constatado en este estudio, son precisamente las prácticas docentes más centradas en los niveles de contenidos de las materias las que alejan más a muchos de estos estudiantes de su grupo clase y del sistema.

Para concluir, creemos conveniente reiterar que el hecho de que este trabajo se base en estudios de caso instrumentales y se haya llevado a cabo siguiendo las orientaciones conceptuales de Stake (2007) cuando habla de estudios de caso colectivos, nos permite "producir generalizaciones proposicionales» (p. 79); lo que no quiere decir que pretendamos generalizar estos hallazgos, ya que, por la propia naturaleza de la metodología planteada y la dimensión singular e intrínseca de todo caso, entraríamos en contradicción epistemológica. En última instancia, es el investigador, docente o lector el que valorará hasta qué punto pueden contribuir estas aportaciones a sus reflexiones o a sus tareas. Ahora bien, la suma y contraste de trabajos de este tipo en ámbitos educativos variados, nos aportará comprensión y, en cuanto sigamos profundizando, serán cada vez más útiles para orientar y fundamentar la toma de decisiones en las políticas y prácticas educativas. Con nuestras interpretaciones y asertos, pretendemos aportar un "grano" de conocimiento en aras a la comprensión de la complejidad educativa en los tiempos multiculturales actuales.

\section{NOTA}

1 La codificación para referir las fuentes en este escrito es la siguiente: Información extraída de los Casos (Cas.1; Cas.2; Cas.3, etc). Grupos de Discusión (gru.dis). Personas/informantes: Profe, Profa (profesor/a); Orient (Orientador/a); Fami (Familiar); Invg (Investigador/a). 


\section{REFERENCIAS BIBLIOGRÁFICAS}

Aguado Odina, M.T. \& Grañeras Pastraña, M. (Eds.). (2010). Diversidad cultural y logros de los estudiantes en educación obligatoria. Lo que sucede en las escuelas. Informe de investigación. Colección de Estudios del CREADE. Madrid: MECD.

Alegre Canos, M. A. (2008). Educación e inmigración. ¿Un binomio problemático? Revista de Educación, 345, 61-82.

Alegre, M. Á., Ricard, B. \& González Motos, S. (2012). Experiencias escolares iniciales del alumnado inmigrado: comienzos que marcan. Educación $X X 1,15(2), 137-158$

Barbour, R. (2013). Los grupos de discusión en la investigación cualitativa. Madrid: Morata.

Castel, R. (1995). De la exclusión como estado a la vulnerabilidad como proceso. Archipiélago, 21, 27-36.

Castel, R. (2007). Los desafíos de las mutaciones sociales, políticas y económicas del siglo XxI. Temas y debates: Revista Universitaria de Ciencias Sociales, 13, 27-39.

CC.OO. (2013). Evolución, de 2008 a 2012, de la tasa de abandono educativo temprano: actividad laboral y formación de los jóvenes en época de crisis. Madrid: Secretaría de Acción SindicalProfesorado Enseñanza Pública.

Colectivo IOÉ (2007). Inmigración, género y escuela: Exploración de los discursos del profesorado y del alumnado. Madrid: CIDE, Ministerio de Educación y Ciencia.

Contreras Domingo, J. (2011). La autonomía del profesorado. Madrid: Morata.

Defensor del Pueblo (2003). La escolarización del alumnado de origen inmi- grante en España: análisis descriptivo y estudio empírico. Madrid: Departamento de Comunicación del Defensor del Pueblo.

Escudero Muñoz, J. M. (2005). Fracaso escolar, exclusión educativa: ¿de qué se excluye y cómo? Profesorado, Revista de Currículum y Formación del Profesorado, 9(1), 1-24.

Escudero Muñoz, J. M. (2009). La Formación del profesorado de Educación Secundaria: contenidos y aprendizajes docentes. Revista de Educación, 350, 79-104.

Espínola Hoffmann, V. \& Claro Stuardo, J. P. (2010). Estrategias de prevención de la deserción en la Educación Secundaria: perspectiva latinoamericana. Revista de Educación, núm. extraordinario, 257-280.

Essomba, M. A. (2006). Liderar escuelas interculturales e inclusivas. Equipos directivos y profesorado ante la diversidad cultural y la inmigración. Barcelona: Graó.

Fernández Larragueta, S. \& Rodorigo, M. (2012). Study of the coordination and effectiveness of the inter-professional consulting system for immigrants» educational inclusión in Andalusian Schools. Procedia-Social and Behavioral Sciences, 46, 2625-2629.

Fernández Sierra, J. (2006). Educación para la carrera y globalización: ¿atrapados en las contradicciones sociolaborales neoliberales? REOP-Revista Española de Orientación y Psicopedagogía, 17(2), 261-272.

Fernández Sierra, J. (2011). Formar para la economía del conocimiento vs, educar para la sociedad del conocimiento. Málaga: Ediciones Aljibe. 
Gimeno Sacristán, J. \& Pérez Gómez A. I. (2008). Comprender y transformar la enseñanza. Madrid: Morata.

Hargreaves, A. \& Fullan, M. (2012). Professional capital: Transforming teaching in every school. New York: Teachers College Press.

Instituto Valenciano de Investigaciones Económicas (IVIE) y Bancaja (2011). El alumnado extranjero en el sistema educativo español. Monográfico. Capital Humano, 123, 1-8

Leiva Olivencia, J.J. (2011). La educación intercultural: un compromiso educativo para construir una escuela sin exclusiones. Revista Iberoamericana de Educación, 56(1), 1-14.

Marí-Klose, P. (Ed.). (2009). Informe de la inclusión social en España 2009. Barcelona: Fundació Caixa Catalunya.

MECD (2012). Datos y cifras. Curso escolar 2011-2012. Madrid: Secretaría General Técnica. Subdirección General de Documentación y Publicaciones.

Mena Martínez, L., Fernández Enguita, M. \& Riviére Gómez, J. (2010). Desenganchados de la educación: procesos, experiencias, motivaciones y estrategias del abandono y del fracaso escolar. Revista de Educación, núm. extraordinario, 119-145.

O’Keefe, P. K.; Westgate, K. \& Wisner, B. (1976). Taking the "naturalness» out of «natural» disasters. Nature, 260 (5552), 566-567.

OCDE (2013). Informe español. Programa Internacional para la Evaluación de los Alumnos (PISA). PISA 2012. Madrid: Ministerio de Educación, Cultura y Deporte. Recuperado de http:// goo.gl/1cr7uA

Pérez de Armiño, K. (2000). Diccionario de Acción Humanitaria y Cooperación al Desarrollo. Barcelona: Icaria.
Pérez Gómez, A. I. (1998). La cultura escolar en la sociedad neoliberal. Madrid: Morata.

Pérez Gómez, A.I. (2012). Educarse en la era digital. Madrid: Morata.

Planella Rivera, J. P. (2008). Educación social, acompañamiento y vulnerabilidad: hacia una antropología de la convivencia. Revista Iberoamericana de Educación, 46(5), 1-14.

RAE (2001). Diccionario de la lengua española. (22. ED.). Recuperado de http://lema.rae.es/drae/

Reimer, E. (1973). La escuela ha muerto: alternativas en materia de educación. Barcelona: Barral.

Roca Cobo, E. (2010). El abandono temprano de la educación y la formación en España. Revista de Educación, núm. extraordinario, 31-62.

Stake, R. E. (2007). Investigación con estudio de casos. Madrid: Morata.

Subirats Humet, J., Gomà Carmona, R. y Brugué Torruella, J. (Eds.). (2005). Análisis de los factores de exclusión social. Bilbao: Fundación BBVA y Institut d»Estudis Autonomics.

Tezanos, J.F. (2001). La sociedad dividida. Estructura de clases y desigualdades en las sociedades tecnológicas. Madrid: Biblioteca Nueva.

Torres Santomé, J. (2008). Diversidad cultural y contenidos escolares. Revista de Educación, 345, 83-110.

Turner, B.L., Kasperson, R. E., Matson, P. A., McCarthy, J. J., Corell, R. W., Christensen, L., \& Schiller, A. (2003). A framework for vulnerability analysis in sustainability science. Proceedings of the national academy of sciences, 100 (14), 8074-8079. 
Vélaz de Medrano, C. (2005). Cómo eludir el rechazo y la exclusión social. Cuadernos de Pedagogía, 348, 58-61.
Vila, I. (2005). ¿Nivel sociocultural o desconocimiento de la lengua? Perspectivas CEP, 8, 23-54. 


\section{PERFIL ACADÉMICO Y PROFESIONAL DEL AUTOR}

Juan Fernández Sierra. Doctor en Ciencias de la Educación. Catedrático del área de Didáctica y Organización Escolar en la Universidad de Almería. Director del Programa de Doctorado Innovación Educativa y del Grupo de Investigación: Asesoramiento, Perfeccionamiento y Calidad de la Enseñanza. Líneas de investigación: Innovación educativa; Política, calidad y evaluación; multiculturalidad; inserción sociolaboral.

Dirección del autor: Facultad de Ciencias de la Educación.

Dpcho. 2.30-A

Universidad de Almería

Carretera Sacramento, s/n

La Cañada de San Urbano

04120 - Almería

E-mail: jfsierra@ual.es

Fecha Recepción del Artículo: 30. Julio. 2014

Fecha modificación Artículo: 24. Octubre. 2014

Fecha Aceptación del Artículo: 13. Noviembre. 2014

Fecha Revisión para Publicación: 06. Julio. 2016 